\title{
The Use of the Maximum Entropy Principle to Approximate Turbulent Probability Density Functions
}

\author{
RW Derksen ${ }^{1}$ \\ ${ }^{1}$ Department of Mechanical Engineering, University of Manitoba, Winnipeg, Canada \\ Robert_Derksen@ad.umanitoba.ca
}

\begin{abstract}
The fundamental problem of turbulence is closing the infinite sequence of equations that result from the application of Reynolds averaging to the governing equations. These equations model the moments of the turbulent probability density function, PDF, such as the first, second, and higher order moments, with each equation depending on higher order moments. The ability to relate the set of moments of order $n$ to moments of order $n+1$ would truncate the sequence of equations resulting in a closed, finite system of equations. Boltzmann showed that the entropy of a thermodynamic state is proportional to the log of the probability of its existence and relates entropy to chaos. This concept was fully developed for information theory by Shannon. The result of Shannon's work is that it results in a constructive criterion to develop probability distributions based on partial knowledge, a type of statistical inference called the maximum entropy principle. This approach was proposed by Jaynes to solve problems in statistical mechanics. It can be argued that the statistical character of the fluctuations found in turbulence should also follow a maximum entropy principle as supported by Townsend's statement that the turbulent fluctuations are an intermediate state between the energy of the flow and ultimately heat. The maximum entropy method determines the PDF that maximizes the entropy subject to several constraints. One method is to use a finite number of lower order moments. This method has a simple solution for single and multiple degrees of freedom. The presentation will review the analytical solutions to the maximum entropy method for both single and multiple degrees of freedom. A review of the comparison of the moments generated from a maximum entropy approximation for a single degree of freedom using data for velocity, skin-friction, and temperature fluctuations. The comparisons are based on constraining the first four measured moments and comparing the computed fifth and sixth moments to the corresponding measured moments. The presentation will give the maximum entropy distribution for multiple degrees of freedom. Additionally, the presentation will discuss the number of degrees of freedom, the number of constraints required, as well as the resultant constraint equations that exist for a turbulent flow.
\end{abstract}

